0 to 2 at discharge. Association of outcomes with prior CMBs was quantified using multiple logistic regression.

Results Among 155 patients included in the analysis (mean age 67.5 , SD 1.15; 56.2\% female), 20 (12.9\%) had CMBs on GRE imaging prior to EVT. Among those with CMBs, 8 $(40 \%)$ patients had strictly lobar, 4 (20\%) had mixed cortical/ subcortical, and $5(25 \%)$ patients had high burden $(>5)$ microbleeds. Older age, female sex, and presence of atrial fibrillation were significantly higher in patients with CMBs. Overall, 41 (26.4\%) developed ICH, among whom 9 (5.8\%) were symptomatic. In multivariable analysis adjusting for age, sex, presenting NIHSS, hypertension, diabetes mellitus, atrial fibrillation, intravenous tPA, and successful recanalization, there was no statistically significant association between the presence of $\mathrm{CMB}$ and ICH (OR 1.19, 95\% CI 0.25-4.90, $\mathrm{p}=0.83$ ) or favorable clinical outcome at discharge (OR 1.25, $95 \%$ CI $0.38-4.01, \mathrm{p}=0.71)$. Results remained unchanged in subgroup analyses based on CMB location or burden.

Conclusion Our analysis indicates that the presence of CMBs is not significantly associated with poor clinical outcome or the risk of ICH following EVT.

Disclosures H. Saber: None. J. Saver: None. V. Szeder: None. M. Nour: None. K. Khatibi: None. L. Ponce Mejia: None. S. Tateshima: 2; C; Medtronic, Stryker, Cerenovus. G. Colby: 2; C; Medtronic, Stryker, MicroVention. N. Kaneko: None. R. Jahan: 2; C; Medtronic. G. Duckwiler: 2; C; Medtronic. D. Liebeskind: 2; C; Medtronic.

\section{P-017 EVALUATION OF RADIOFREQUENCY-INDUCED HEATING IN AN X-RAY AND MR-VISIBLE INTERVENTIONAL CATHETER AT $3.0 \mathrm{~T}$}

${ }^{1}$ B Kilbride* ${ }^{1} \mathrm{~J}$ Massachi, ${ }^{2} \mathrm{~S}$ Ahn, ${ }^{2} \mathrm{~K}$ Mueller, ${ }^{3} \mathrm{C}$ Jordan, ${ }^{4} \mathrm{~A}$ Losey, ${ }^{1} \mathrm{~A}$ Martin, ${ }^{1} \mathrm{~T}$ Moore, ${ }^{1} \mathrm{M}$ Wilson, 'S Hetts. 'Radiology and Biomedical Imaging, University of California San Francisco, San Francisco, CA; ${ }^{2}$ Siemens Medical Solutions, Malvern, PA; ${ }^{3}$ Texas AandM University, Houston, $T X_{i}^{4}$ Temple University, Philadelphia, PA

\subsection{6/neurintsurg-2021-SNIS.53}

Introduction Successful mechanical thrombectomy in the case of acute ischemic stroke can be inferred from the angiogram, and MRI information can provide insight regarding extent of cerebral infarct and whether further reperfusion therapy may increase the risk of hemorrhage. Recently, it has been shown that intra-procedural MRI can influence critical decision making to pursue further endovascular therapy. These decisions have been enabled by combined X-ray angiography MRI suites; all the while compatible tooling has lagged. Here, we have performed initial safety tests in vitro to validate the safety of a polymer-based guide catheter with in-wall X-ray and MR-visible markers.

Methods A guide catheter prototype was built using polymerbased filament in a catheter fabrication facility (Penumbra, Inc., Alameda, CA). Four circumferential marker bands were painted $2 \mathrm{~cm}$ from the distal tip. Markers were an epoxybased radiopaque ink (Creative Materials, Ayer, MA) doped with iron(III) oxide $\left(\mathrm{Fe}_{2} \mathrm{O}_{3}\right)$ nanoparticles of 20-40 nm diameter (Alfa Aesar, Tewksbury, MA). The device was then laminated with a multi-durometer thermoplastic jacket. Experiments were performed in a clinical biplane x-ray neuroangiography and 3.0 T MRI suite (Magnetom Skyra, Siemens Healthineers, AG, Forccheim, Germany).

The catheter was embedded into an acrylic phantom, per ASTM F2182-19e2. The head portion was made of a polyacrylamide gel and the body was filled with saline. The catheter extended $25 \mathrm{~cm}$ into the head and was laterally positioned $17 \mathrm{~cm}$ from the midline of the body and $6 \mathrm{~cm}$ from the midline of the head. Radiofrequency-induced heating of the prototype was assessed for worst-case heating as a function of insertion length. Heating directly adjacent to the catheter was measured using fluoroptic temperature probes (FOT Lab Kit with STF probes; LumaSense, Santa Clara, California). Two probes were embedded at the distal tip of the catheter and $10 \mathrm{~cm}$ proximal to the tip. Temperature was continuously measured over the course of 6 minutes: 2 minutes of baseline prior to imaging, 2 minutes of imaging using a True FISP sequence with a SAR of $4 \mathrm{~W} / \mathrm{kg}$, and 2 minutes of equilibration after imaging. The immersed length started at $95 \mathrm{~cm}$ and was shortened by $5 \mathrm{~cm}$ after each 6 -minute test until the immersed length was $60 \mathrm{~cm}$.

Results Maximum temperature and change in temperature were recorded. The largest temperature change occurred at $95 \mathrm{~cm}$, where the temperature rose by $0.45{ }^{\circ} \mathrm{C}$ and $0.70{ }^{\circ} \mathrm{C}$ at the device tip and $10 \mathrm{~cm}$ proximal to the tip, respectively. Over the entire testing period, the local temperature around the device tip and $10 \mathrm{~cm}$ proximal to the tip rose $1.48^{\circ} \mathrm{C}$ and $1.68^{\circ} \mathrm{C}$, respectively.

Conclusions Initial immersion tests suggest that the worst-case scenario for RF-induced heating of our guide catheter prototype occurs at $95 \mathrm{~cm}$. During the period of our tests, our prototype remained safely under the maximum permitted temperature rise of $+2^{\circ} \mathrm{C} / \mathrm{hr}$ of exposure. This study suggests that our guide catheter may be retained during an intra-procedural MRI without the safety risk of RF-induced device heating over time to the patient.

Disclosures B. Kilbride: None. J. Massachi: None. S. Ahn: None. K. Mueller: None. C. Jordan: None. A. Losey: None. A. Martin: None. T. Moore: None. M. Wilson: None. S. Hetts: None.

\section{P-018 VESSEL WALL IMAGING WITH MRI TO ASSESS ENDOTHELIAL WALL INJURY POST MECHANICAL THROMBECTOMY}

${ }^{1}$ R Mattay*, ${ }^{2} \mathrm{C}$ Favilla, ${ }^{1} \mathrm{~B}$ Pukenas, ${ }^{3} \mathrm{O}$ Choudhri, ${ }^{1} \mathrm{R}$ Hurst, ${ }^{1} \mathrm{~S}$ Nabavizadeh. ${ }^{1}$ Radiology, Hospital of the University of Pennsylvania, Philadelphia, PA; ${ }^{2}$ Neurology, Hospital of the University of Pennsylvania, Philadelphia, PA; ${ }^{3}$ Neurosurgery, Hospital of the University of Pennsylvania, Philadelphia, PA

\subsection{6/neurintsurg-2021-SNIS.54}

Purpose Mechanical thrombectomy using stent retrievers, aspiration catheters, or a combination of both are commonly used in practice to treat large vessel occlusion. Recent MR Vessel Wall Imaging (VWI) studies in patients status post thrombectomy in the acute and subacute phases have shown persistent increased vessel wall enhancement when compared to controls, which is likely representative of the endothelial damage to the vessel wall as shown on earlier histopathologic studies. In this study of 10 patients who underwent thrombectomy at our institution, we preliminarily explore the type and amount of vessel wall enhancement associated with different methods of thrombectomy and number of passes.

Materials and Methods 10 adult patients from 5/7/2019 to 9/ 9/2020 with occlusion of M1 segment who were admitted following mechanical thrombectomy underwent a $3 \mathrm{~T}$ brain MRI within 48 hours post-thrombectomy which included a 3D T1 black blood sequence before and after intravenous 


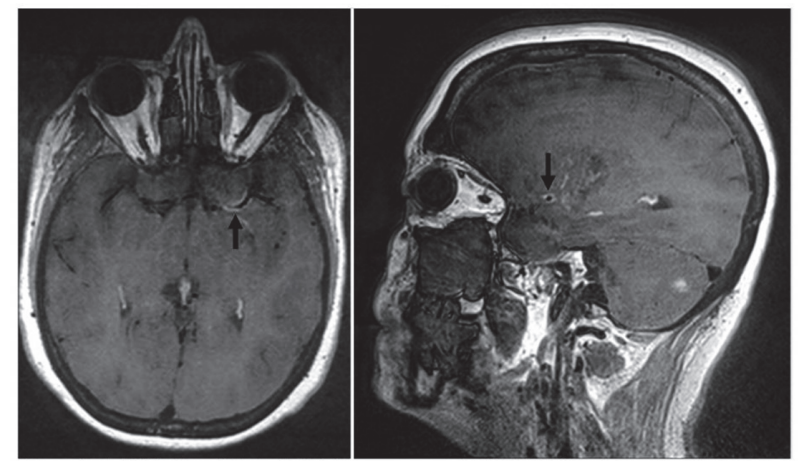

Abstract P-018 Figure 1 Axial (left) and Sagittal (right) post-contrast 3D T1 black blood sequence showing circumferential vessel wall enhancement of the left M1 segment in a patient who recently received mechanical thrombectomy with three passes of the stent retriever

gadolinium. Enhancement data variables from the MRI that were recorded included pattern (circumferential versus eccentric) and amount of maximal contrast enhancement based on region of interest differences in the region of highest T1 signal between post and pre-contrast T1 sequences. Following thrombectomy, the type and number of passes with each device was recorded.

Results 10 adult patients (6 F, Mean age of 65.7 years) were included in this study. Two received stent retriever with aspiration catheter, 7 received stent retriever only, and 1 received aspiration only. All 10 patients demonstrated circumferential enhancement in the region of retrieved clot, including the one patient who received aspiration only. Of the 9 patients that received the stent retriever, recanalization was achieved in 1 pass in 4, while in the other 5 patients, recanalization was achieved after multiple passes (range 2-4 passes). Dichotomizing the data between 1 and multiple passes, there was a difference in the amount of mean maximal enhancement of the involved vessel wall (single pass: 75, Std Dev 28.39 vs multiple passes: 110.6, Std Dev 51.75), although this did not reach statistical significance due to a small number of patients.

Conclusions All patients showed circumferential wall enhancement despite type of thrombectomy technique, suggesting that all techniques may be associated with a similar mechanism of endothelial wall injury. Moreover, those patients who required multiple passes of the stent retriever for recanalization demonstrated a higher mean maximal enhancement when compared to those patients who required just one pass. Further exploration with an increased number of patients is warranted.

Disclosures R. Mattay: None. C. Favilla: None. B. Pukenas: None. O. Choudhri: None. R. Hurst: None. S. Nabavizadeh: None.

\section{P-019 CLINICAL SIGNIFICANCE OF CONTRAST STAINING AFTER MECHANICAL THROMBECTOMY FOR ACUTE ISCHEMIC STROKE}

T Lazaro*, P Cotton, C English, K Katlowitz, O Tanweer, D Raper. Neurosurgery, Baylor College of Medicine, Houston, TX

\subsection{6/neurintsurg-2021-SNIS.55}

Background Acute ischemic stroke is a leading cause of mortality and morbidity throughout the world. Thrombolysis with intravenous tissue plasminogen activator (tPA) is often the first-line therapy after presentation. For select patients with large vessel occlusions (LVO), mechanical thrombectomy (MT) is the gold standard to achieve cerebral reperfusion, in which a variety of clot retriever devices and aspiration catheters are employed to remove the clot. After MT, a computed tomography (CT) scan of the head is often done shortly after to evaluate for stroke progression and hemorrhagic transformation; however, in some patients, contrast can remain within the parenchyma of the brain, referred to as contrast staining (CS). While an unclear radiologic finding, previous studies have suggested that it represents injury to small vessels branching from the occluded vessel during thrombectomy (Renu et al. Stroke. 2014). As a result, CS has been associated with a higher risk intracerebral hemorrhage $(\mathrm{ICH})$ after $\mathrm{MT}$.

Objective In this study, we look to (1) better characterize the clinical significance of post-MT CS in our cohort of acute ischemic stroke patients and (2) determine whether specific devices deployed at the time of thrombectomy are correlated with CS.

Methods We retrospectively reviewed patients from 2017-2020 who underwent MT. Included patients were those that presented within a LVO (i.e. clot within the internal carotid artery, proximal anterior cerebral artery, proximal middle cerebral artery, vertebral artery, or basilar artery) and underwent MT within 24 hours of symptom onset. Patients that had vessel occlusions related to prior surgical or endovascular procedures were excluded. Clinical outcomes were reported with the modified Rankin Scale (mRS).

Results Patients with CS were diagnosed with early ICH (diagnosed within 24 hours after MT) more often that patients without CS $(59 \%$ vs $18 \%$, respectively; p < 0.001$)$. The groups did not differ in late ICH (diagnosed after 24 hours). Patients with symptomatic ICH had higher $\mathrm{mRS}$ at discharge (median of 5 vs 3 , respectively; $p=0.02$ ). However, CS was not independently associated with worsened mRS after MT $(\mathrm{p}=0.47)$. In addition, larger diameter clot retriever devices and catheters were not significantly correlated with the presence of contrast staining or development of symptomatic ICH.

Conclusion Contrast staining is associated with the development of ICH, particularly in the early period after MT and worse clinical outcomes. While patients with $\mathrm{ICH}$ had worse functional outcomes at discharge, contrast staining itself was not associated with worse outcomes. Lasty, larger diameter clot retriever devices and catheters were not significantly correlated with the presence of contrast staining.

Disclosures T. Lazaro: None. P. Cotton: None. C. English: None. K. Katlowitz: None. O. Tanweer: None. D. Raper: None.

\section{P-020 PROCESS IMPROVEMENT WITH PRE-TRANFSER INTUBATION FOR PATIENTS UNDERGOING MECHANICAL THROMBECTOMY; NOT JUST FOR THE COVID PANDEMIC}

\footnotetext{
${ }^{1,2} \mathrm{M}_{\text {Stiefel }}{ }^{*},{ }^{1,2} \mathrm{~J} \mathrm{Strauss,}^{3} \mathrm{~J}$ Shapiro, ${ }^{3} \mathrm{~S}$ Solomon, ${ }^{2} \mathrm{C}$ Lytle, ${ }^{2} \mathrm{~T}$ Esdale, ${ }^{1,2} \mathrm{~A}$ Schuette. ${ }^{1}$ Cerebrovascular and Endovascular Neurosurgery, Piedmont Healthcare, Atlanta, GA; ${ }^{2}$ Piedmont Stroke Center, Atlanta, GA; ${ }^{3}$ Anesthesia, Piedmont Healthcare, Atlanta, GA
}

10.1136/neurintsurg-2021-SNIS.56 